Bentler

Grundsätze ordnungsmäßiger Bilanzierung für die Equitymethode 
Bochumer Beiträge

zur Unternehmungsführung

und Unternehmensforschung

Herausgegeben von

Prof. Dr. Hans Besters

Prof. Dr. Dr. h. c. Walther Busse von Colbe

Prof. Dr. Werner Engelhardt

Prof. Dr. Arno Jaeger

Prof. Dr. Gert Laßmann

Prof. Dr. Wolfgang Maßberg

Prof. Dr. Eberhard Schwark

Prof. Dr. Rolf Wartmann

Band 40

Institut für Unternehmungsführung

und Unternehmensforschung

der Ruhr-Universität Bochum

Eine Aufstellung der in dieser Schriftenreihe erschienenen Bände finden Sie am Ende des Buches. 
Martin Bentler

\section{Grundsätze ordnungsmäßiger Bilanzierung für die Equitymethode}


CIP-Titelaufnahme der Deutschen Bibliothek
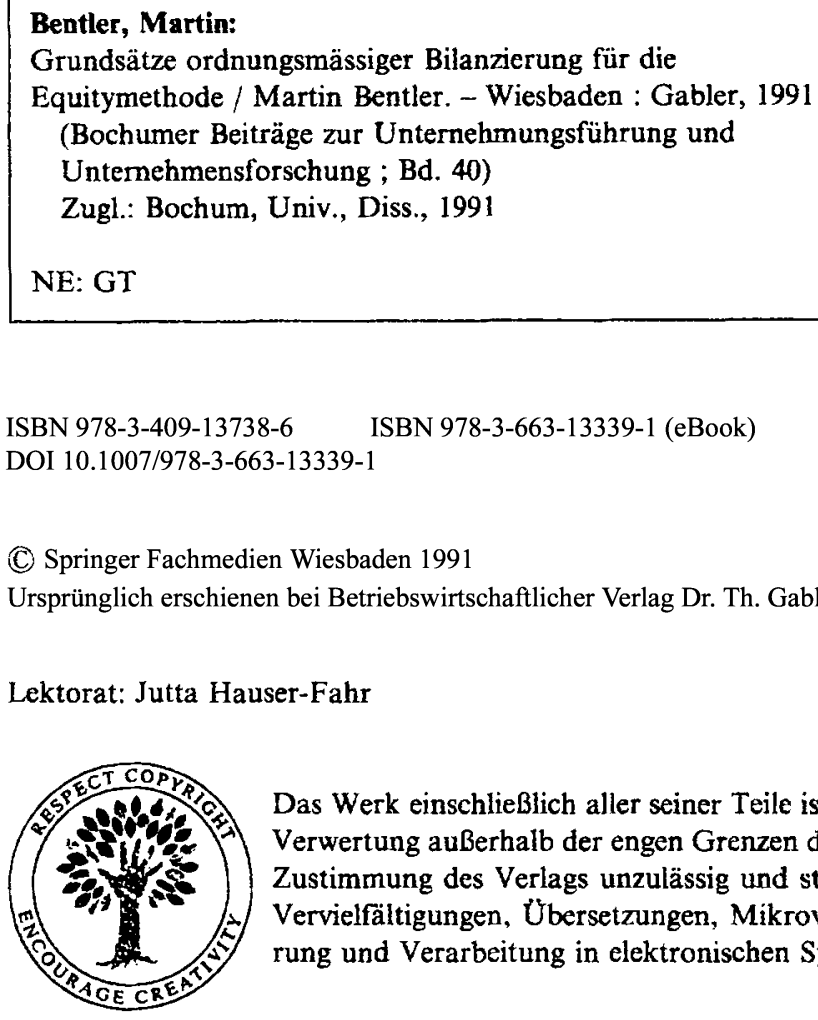

Das Werk einschließlich aller seiner Teile ist urheberrechtlich geschützt. Jede Verwertung außerhalb der engen Grenzen des Urheberrechtsgesetzes ist ohne Zustimmung des Verlags unzulässig und strafbar. Das gilt insbesondere für Vervielfältigungen, Übersetzungen, Mikroverfilmungen und die Einspeicherung und Verarbeitung in elektronischen Systemen.

Höchste inhaltliche und technische Qualität unserer Produkte ist unser Ziel. Bei der Produktion und Verbreitung unserer Bücher wollen wir die Umwelt schonen: Dieses Buch ist auf säurefreiem und chlorfrei gebleichtem Papier gedruckt. Die Einschweißfolie besteht aus Polyäthylen und damit aus organischen Grundstoffen, die weder bei der Herstellung noch bei der Verbrennung Schadstoffe freisetzen.

Die Wiedergabe von Gebrauchsnamen, Handelsnamen, Warenbezeichnungen usw. in diesem Werk berechtigt auch ohne besondere Kennzeichnung nicht zu der Annahme, daß solche Namen im Sinne der Warenzeichen- und Markenschutz-Gesetzgebung als frei zu betrachten wären und daher von jedermann benutzt werden dürften. 
Geleitwort

Im angelsächsischen Raum ist die Equitymethode zur Bewertung von Beteiligungen seit langem üblich. Für die deutsche Bilanzierungspraxis stellt sie weitgehend Neuland dar. Seit dem 1.1.1990 ist die Ubergangsfrist zur Anwendung des neuen Bilanzrechts auf den Konzernabschluß abgelaufen. Bei Vorliegen der entsprechenden Kriterien ist die Equitybewertung damit auch in Deutschland obligatorisch.

Die vorliegende Arbeit kommt aus der Praxis. Der Verfasser war während der Untersuchung in einem großen deutschen Konzern der Elektroindustrie u.a. mit der Implementierung der Equitymethode betraut und konnte deshalb unmittelbar die in der Praxis auftretenden Probleme einbringen. Die systematische Darstellung der Equitymethode wird durch umfangreiche Beispielrechnungen auf der Grundlage publizierter Weltabschlußdaten eines großen deutschen Konzerns der Autoindustrie anschaulich ergänzt.

In der Bilanzierungspraxis auftretende Probleme bei der Equitybewertung werden zunächst beschrieben und analysiert. Im nächsten Schritt diskutiert der Autor unterschiedliche mögliche Problemlösungen und versucht anschließend, zu jedem Einzelfall die betriebswirtschaftlich sinnvollste Lösungsalternative unter Rückgriff auf die allgemeinen Grundsätze ordnungsmäßiger Bilanzierung abzuleiten. Die Untersuchung schließt dabei auch die Problemkreise assoziierter Unternehmen im Ausland und der Beendigung der Equitymethode ein. Sie enthält außerdem einen Vorschlag zur konkreten Ausgestaltung einer standardisierten Nebenrechnung. Dafür entwickelt der Verfasser einen detaillierten Leitfaden zur Equitybewertung für den Bilanzierungspraktiker. 
Vorwort

Die vorliegende Arbeit wurde im Sommersemester 1991 von der Fakultät für Wirtschaftswissenschaft der Ruhr-Universität Bochum als Dissertation angenommen.

Meinem Doktorvater, Herrn Prof. Dr. Dr. h.c. Walther Busse von Colbe, danke ich aufrichtig, daß er die Arbeit als externe Promotion akzeptiert, wissenschaftlich betreut und mit vielen wertvollen Hinweisen und Anregungen unterstützt hat. Herrn Prof. Dr. Klaus Chmielewicz danke ich für die Ubernahme des Korreferats. Dem Direktorium des Instituts für Unternehmungsführung und Unternehmensforschung der Ruhr-Universität und dem Gabler Verlag bin ich für die Aufnahme der Arbeit in diese Schriftenreihe zu Dank verpflichtet.

Einige Kollegen aus der Praxis, besonders die Herren Hans-Albrecht Garbe und Klaus Kramer sowie mein direkter Vorgesetzter Herr Bernd Vogt, haben mit kritischen Armerkungen zu vielen Problemen und durch ihre ständige Diskussionsbereitschaft wesentlich zum Gelingen dieser Arbeit beigetragen. Innen - sowie Herrn Dr. Bernhard Pellens von der Ruhr-Universität - sei an dieser Stelle herzlich gedankt.

Eine wesentliche Hilfe leistete Frau Renate Steffes'enn-Witte, die den ersten Entwurf sowie die diversen Uberarbeitungen in teilweise atemberaubender ceschwindigkeit in eine maschinengeschriebene Form brachte. Inr, und last but not least meinem Vater, Herrn Günther Bentler, der mich beim Korrekturlesen sämtlicher Fassungen kontinuierlich unterstützte, gilt mein besonderer Dank. 
Geleitwort von Prof. Dr. Dr. h.c. Walther Busse von Colbe

Abkürzungsverzeichnis

Abbildungsverzeichnis

Ubersichtsverzeichnis

Anlagenverzeichnis

Erstes Kapitel: Problemstellung und Aufbau der Arbeit

I. Problemstellung

II. Aufbau der Arbeit

Zweites Kapitel: Grundlagen

I. Funktionen des Konzernabschlusses 7

A. Informationsfunktion 7

B. Dokumentations- und Zahlungsbemessungsfunktion

II. Struktur und Ziel der Equitymethode

III. Grundsätze ordnungsmäßiger Konzernrechnungslegung

A. Uberblick

B. Ausgewählte Grundsätze

1. Fiktion der rechtlichen Einheit des Konzerns

2. Einheitliche Bewertung und Anwendung der Vollkonsolidierungsgrundsätze

3. Realisationsprinzip

4. Stetigkeit

5. Bilanzkongruenz

6. Wesentlichkeit

7. Wirtschaftlichkeit 27

8. Zusammenfassung 29

Drittes Kapitel: Abgrenzung der relevanten Beteiligungen 30

I. Assoziierte Unternehmen $\quad 30$

II. Gemeinschaftsunternehmen 34

III. Konzernunternehmen $\quad 35$

IV. Zusammenfassung 
Viertes Kapitel: Struktur und Ausprägungen des Verfahrens gemäß $\S 312$ HGB

I. Grundstruktur 43

A. Neubewertung im Erwerbszeitpunkt 44

B. Zwischenerfolgseliminierung 48

1. Gesetzliche Grundlagen 48

2. Quotale oder volle Eliminierung 49

3. Eliminierung $u p$ - und/oder downstream 51

4. Technik 53

C. Steuerabgrenzung 54

1. Grundlagen der Bilanzierung latenter Steuern 54

2. Latente Steuern dem Grunde nach bei Equitybewertung 58

a. Problematik der Anwendung von $\S 306$ HGB auf die Equitymethode 58

b. Fortschreibung aus Ergebnis 59

c. Auflösung von Reserven und Lasten, Goodwill und
Badwill

d. Zwischenerfolgseliminierung 63

3. Latente Steuern der Höhe nach 64

D. Mögliche Verfahrensausprägungen aufgrund von Zwischenerfolgseliminierung und Steuerabgrenzung 67

$\begin{array}{ll}\text { E. Zusammenfassung } & 70\end{array}$

II. Bilanzierung im Erwerbszeitpunkt 72

A. Bestimmung der Anschaffungskoster, 72

B. Abgrenzung des erworbenen Eigenkapitals 73

1. Im Einzelabschluß 73

2. Im Konzernabschluß 76

3. Relevanter Abschluß der Beteiligungsgesellschaft 79

4. Relevante Beteiligungsquoten 82

5. Minderheitenausweis bei Equitybewertung 85

C. Anfangsdifferenz 86

1. Umbewertung von Bilanzpositionen 86

2. Geschäftswert 92 
Seite

III. Fortschreibung in den Folgejahren 93

A. Regelmäßige Fortschreibung 93

1. Ergebnis 93

2. Ausschüttung 97

3. Abschreibung einer aktivischen Anfangsdifferenz 99

4. Auflösung einer passivischen Anfangsdifferenz 102

5. Zwischenerfolgseliminierung 104

$\begin{array}{ll}\text { 6. Steuerabgrenzung } & 107\end{array}$

7. Erfolgsneutrale Eigenkapitalveränderungen 108

B. Außerordentliche Fortschreibung 111

1. Zugang 112

a. Quotenneutral 113

b. Quotenwirksam 114

2. Abgang 115

a. Quotenneutral 116

b. Quotenwirksam 119

3. Spezialfälle 121

a. Quotenverminderung ohne Anteilsverkauf 122

b. Quotenerhöhung ohne Anteilszukauf 123

c. Asymmetrische Gewinnverteilung 124

4. Abschreibungen und Zuschreibungen 125

$\begin{array}{ll}\text { IV. Zusammenfassung } & 127\end{array}$

Fünftes Kapitel: Aufbau der Nebenrechnung und Beispiele 131

I. Aufbau der Nebenrechnung 131

II. Beispielrechnungen auf Basis von Daten eines deutschen
Großunternehmens

A. Grundbeispiel (Beispiel 1) 138

1. Zeitgleiche Bilanzierung (Beispiel 1.1) 138

2. Zeitversetzte Bilanzierung (Beispiel 1.2) 154

B. Eliminierung von Zwischenerfolgen (Beispiel 2) 163

C. Passiver Unterschiedsbetrag bei Erwerb (Beispiel 3) 172 


\section{Seite}

Sechstes Kapitel: Bilanzierung in Ersteinbeziehungszeitpunkt

I. Ubergang auf neues Recht (Beispiel 4)

II. Sukzessiver Erwerb (Beispiel 5)

195

III. Zusammenfassung

\section{Siebentes Kapitel: Assoziierte Unternehmen im Ausland}

I. Vereinheitlichungen in Ansatz und Bewertung 204

$\begin{array}{ll}\text { II. Währungsumrechnung } & 210\end{array}$

A. Einheitliche Umrechnung zum Stichtagskurs 213

B. Umrechnung mit differenzierten historischen Kursen 215

$\begin{array}{ll}\text { C. Spezifika bei Anwendung der Equitymethode } & 217\end{array}$

$\begin{array}{ll}\text { 1. Umrechnung im Erwerbszeitpunkt } & 217\end{array}$

2. Umrechnung in den Folgeperioden 218

$\begin{array}{ll}\text { a. Jahresüberschuß } & 218\end{array}$

b. Jahresfehlbetrag 219

c. Auflösung der Anfangsdifferenz 221

d. Dividende 222

e. Zugänge und Abgänge 224

f. Zwischenerfolge 224

g. Steuerabgrenzung 226

h. Sonstige Anpassungen 227

i. Abschreibungen und Zuschreibungen 228

j. Niederstwerttest 229

3. Modifikation von Beispiel 2 (Beispiel 22) 230

D. Umrechnung im Ersteinbeziehungszeitpunkt 243

1. Ubergang auf neues Recht 243

2. Sukzessiver Erwerb 244

E. Hochinflationsländer 245

F. Zusammenfassung 248 
Seite

Achtes Kapitel: Beendigung der Equitymethode

I. Verkauf einer "at equity" angesetzten Beteiligung

II. Rückkehr zur Anschaffungskostenmethode

III. Ubergang zur Vollkonsolidierung

Neuntes Kapitel: Ausweis in Bilanz, Gewinn- und Verlustrechnung und Anlagenspiegel

Zehntes Kapitel: Zusarmenfassung

Anlagen

Verzeichnis der Geschäftsberichte 


\section{Abkürzungsverzeichnis}

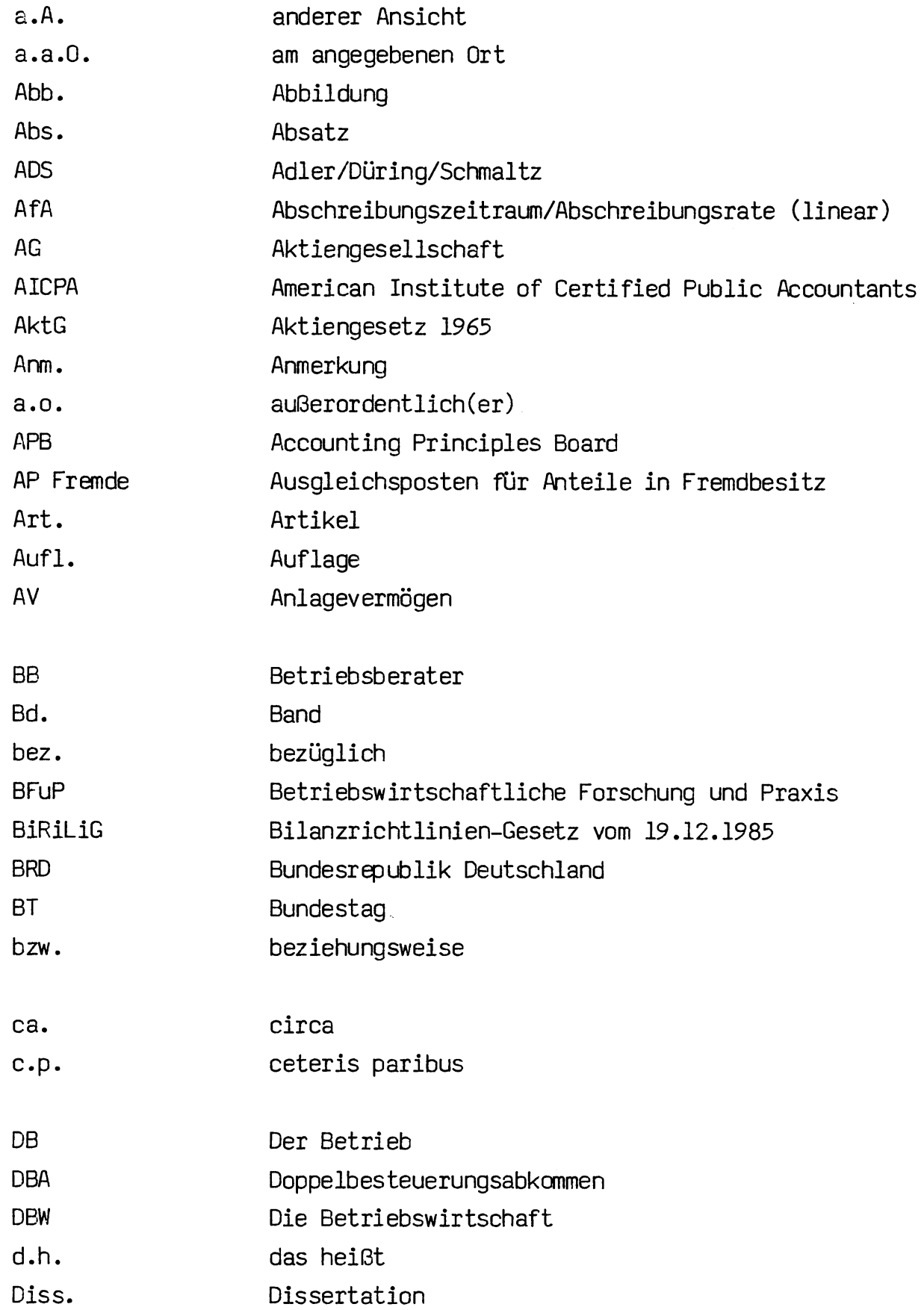




$\begin{array}{ll}\text { EAV } & \begin{array}{l}\text { Ergebnisabführungsvertrag } \\ \text { EG }\end{array} \\ \text { EGHGB } & \begin{array}{l}\text { Einführungsgesetz zum Handelsgesetzbuch } \\ \text { (Übergangsvorschriften) }\end{array} \\ \text { EK } & \begin{array}{l}\text { Eigenkapital } \\ \text { Equitymethode }\end{array} \\ \text { EStG } & \text { Einkommensteuergesetz } \\ \text { f., ff. } & \text { folgende, fort folgende } \\ \text { FASB } & \text { Financial Accounting Standards Board } \\ \text { FAV } & \text { Finanzanlagevermögen } \\ \text { FIFO } & \text { first in first out } \\ \text { Fn. } & \text { Fußnote } \\ \text { FRF } & \text { Französische Francs }\end{array}$

Gesellschaft für Finanzwirtschaft in der Unternehmensführung $\mathrm{e} . \mathrm{V}$.

ggf. gegebenenfalls

GJ Geschäftsjahr

$\mathrm{GmbH}$ Gesellschaft mit beschränkter Haftung

GoB Grundsätze ordnungsmäßiger Buchführung

GoK Grundsätze ordnungsmäßiger Konzernrechnungslegung

GuV Gewinn- und Verlustrechnung

HB II

$\mathrm{HdK}$

$\mathrm{HdR}$

HFA

HGB

Hrsg.

HURB

HWB

HWR

HWReV
Handelsbilanz(en) II

Handbuch der Konzernrechnungslegung

Handbuch der Rechnungslegung

Haupt fachausschuß

Handelsgesetzbuch

Herausgeber

Handwörterbuch unbestimmter Rechtsbegriffe im Bilanzrecht des $\mathrm{HGB}$

Handwörterbuch der Betriebswirtschaft

Handwörterbuch des Rechnungswesens

Handwörterbuch der Revision 


\begin{tabular}{|c|c|}
\hline i.a. & im allgemeinen \\
\hline IASC & International Accounting Standards Committee \\
\hline i.d.R. & in der Regel \\
\hline IDW & Institut der Wirtschaftsprüfer in Deutschland e.v. \\
\hline incl. & inklusive \\
\hline insb. & insbesondere \\
\hline i.s.v.(d.) & im Sinne von (der, des) \\
\hline i.v.m. & in Verbindung mit \\
\hline i.w. & im wesentlichen \\
\hline JF & Jahresfehlbetrag \\
\hline Jg. & Jahrgang \\
\hline JoA & Journal of Accountancy \\
\hline JU & Jahresüberschuß \\
\hline KSt & Körperschaftsteuer \\
\hline kum. & kumuliert \\
\hline LIFO & last in first out \\
\hline lt. & laut \\
\hline MA & Management Accounting \\
\hline No., $\mathrm{Nr}$. & Nummer \\
\hline $\mathrm{OG}$ & Obergesel lschaft \\
\hline 0.9 . & oben genannt \\
\hline p.a. & per anno \\
\hline Par., § & Paragraph \\
\hline PC & Personal-Computer \\
\hline p.r.t. & pro rata temporis \\
\hline
\end{tabular}


RHB

$\mathrm{Rn}$.

S.

SAV

SEC

s.o.

sog.

Sp.

TDM

TFRF

Tz.

u.a.

u.ä.

UG

USA

UV

VAG

VB

vgl.

v.H., \%

$\mathrm{Vj}$.

WPg

WU

z.B.

ZfB

$\mathrm{ZfbF}$

$Z$ fhF

ZGR

Ziff.

Z.T.

$z . z$.
Roh-, Hilfs- und Betriebsstoffe

Randnummer

Seite( $n)$

Sachanlagevermögen

Securities and Exchange Commission

siehe oben

sogenannte $(r)$

Spalte $(n)$

Tausend deutsche Mark

Tausend französische Francs

Textziffer

unter anderem/unter anderen/und andere

und ähnliches

Untergesellschaft

United States of America

Umlaufvermögen

Volkswagen Aktiengesellschaft

Verbindlichkeiten

vergleiche

vom Hundert

Vorjahr

Die Wirtschaftsprüfung

Währungsunterschied

zum Beispiel

Zeitschrift für Betriebswirtschaft

Zeitschrift für betriebswirtschaftliche Forschung

Zeitschrift für handelswissenschaftliche Forschung

Zeitschrift für Unternehmens- und Gesellschaftsrecht Ziffer

zum Teil

zur Zeit 
Abb. 1:

Grundstaffel

Abb. 2:

HGB-Staffel

Abb. 3:

Beispiel zum Buchwert- und Ergebnisspektrum gem. § $312 \mathrm{HGE}$

Abb. 4:

Aufbau der Nebenrechnung

Abb. 5:

Eigenkapitalentwicklung des Beispiel-Konzerns im Betrachtungszeitraum

Abb. 6:

Daten zum Grundbeispiel im Uberblick

Abb. 7:

Zwischenerfolgseliminierung und Steuerabgrenzung

Abb. 8:

Anlagenspiegel zu Beispiel 1.I

\section{Ubersichtsverzeichnis}

Übersicht 1:

Abgrenzung der für die Equitybewertung relevanten Beteiligungen

Ubersicht 2:

Notwendige Buchungen in Beispiel 1.1

\section{Anlagenverzeichnis}

Anlage 1:

Beispiel für die Satzung einer Altersfürsorgegesellschaft

Anlage 2:

Auswertung von Geschäftsberichten 